\title{
A Kinetic Transport Model for the ELMO Bumpy Torus
}

\author{
E. F. Jaeger \\ C. L. Hedrick \\ J. S. Tolliver
}




\section{DISCLAIMER}

This report was prepared as an account of work sponsored by an agency of the United States Government. Neither the United States Government nor any agency Thereof, nor any of their employees, makes any warranty, express or implied, or assumes any legal liability or responsibility for the accuracy, completeness, or usefulness of any information, apparatus, product, or process disclosed, or represents that its use would not infringe privately owned rights. Reference herein to any specific commercial product, process, or service by trade name, trademark, manufacturer, or otherwise does not necessarily constitute or imply its endorsement, recommendation, or favoring by the United States Government or any agency thereof. The views and opinions of authors expressed herein do not necessarily state or reflect those of the United States Government or any agency thereof. 


\section{DISCLAIMER}

Portions of this document may be illegible in electronic image products. Images are produced from the best available original document. 


\section{Printed in the United States of America. Available from}

National Technical Information Service

U.S. Department of Commerce 5285 Port Royal Road, Springfield, Virginia 22161

Price: Printed Copy $\$ 4.50$; Microfiche $\$ 3.00$

This report was prepared as an account of work sponsored by an agency of the United States Government. Neither the United States Government nor any agency thereof, nor any of their employees, contractors, subcontractors, or their employees, makes any warranty, express or implied, nor assumes any legal liability or responsibility for any third party's use or the results of such use of any information, apparatus, product or process disclosed in this report, nor represents that its use by such third party would not infringe privately owned rights. 
Contract No. W-7405-eng-26

FUSION ENERGY DIVISION

A KINETIC TRANSPORT MODEL FOR THE

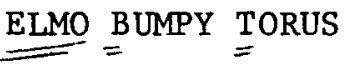

E. F. Jaeger

C. L. Hedrick

J. S. Tolliver

Date Published - May, 1978

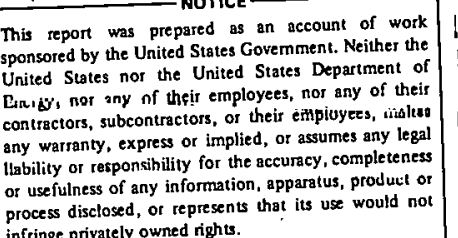

infringe privately owned nights.

NOTICE This document contains information of a preliminary nature. It is subject to revision or correction and therefore dues inot reprosont a final report.

Prepared by the

OAK RIDGE NATIONAL LABORATORY

Oak Ridge, Tennessee 37830

operated by

UNION CARBIDE CORPORATION

for the

DEPARTMENT OF ENERGY 
THIS PAGE

WAS INTENTIONALLY

LEFT BLANK 
Abstract .......................... . v

1. Introduction ....................... 1

2. The Drift Kinetic Equation for EBT . . . . . . . . . 2

3. Simple Analytic Results for An Unperturbed Distribution

Function Which Is Maxwellian . . . . . . . . . . . 7

4. The Collision Operator . . . . . . . . . . . . 8

4.1 Coulomb Collisions . . . . . . . . . . . . . 8

4.2 Ionization and Charge-Exchange Collisions . . . . . . . . 9

4.3 Microwave Collisions . . . . . . . . . . . . 11

5. Results for An Unperturbed Distribution Function Which

Is Maxwellian . . . . . . . . . . . . . . 13

5.1 Numerical Methods . . . . . . . . . . . 13

5.2 Neoclassical Fluxes and Diffusion Rates . . . . . . 14

Conclusions . . . . . . . . . . . . . . . . . 23

Acknowledgment . . . . . . . . . . . . . . . . . 23

References . . . . . . . . . . . . . . . . 24 
THIS PAGE

\section{WAS INTENTIONALLY \\ LEFT BLANK}


A bounce-averaged drift kinetic equation is solved for the toroidal plasma in the ELMO Bumpy Torus (EBT). The distribution function is assumed isotropic in pitch angle and calculated as a function of radius and speed using finite differences on a two-dimensional grid. A Fokker-Planck representation of the collision operator includes Coulomb, microwave, ionizing, and charge-exchange collisions. Ion and electron fluxes, computed as integrals of the distribution function, are of comparable magnitude for ambipolar potentials which are approximately self-consistent. Initial results assume an unperturbed distribution function which is Maxwellian; however, this is not a necessary assumption in the model. Careful accounting of loss regions where electric and magnetic poloidal drifts cancel (super banana particle orbits) leads to ion loss rates which are in some cases two orders of magnitude greater than electron rates. In these cases, radially inward pointing self-consistent electric fields occur with potentials on the order of a few times the ion temperature. These negative field results are in approximate agreement with experiment and appear to be stable to the electric field runaway encountered in positive field cases. 


\section{INTRODUCTION}

The microwave heated ELMO Bumpy Torus (EBT) plasmal is characterized by radially inward pointing ambipolar electric fields ${ }^{2}$ in the stable toroidal or $\mathrm{T}$-mode of operation. Both zero-dimensional point mode $1 \mathrm{~s}^{3,4}$ and one-dimensional fluid transport simulations ${ }^{5}$ have been used to model the toroidal core plasma in EBT with a self-consistent radial electric field. These models solve a series of moments of the bounce-averaged drift kinetic equation ${ }^{6}$ in either zero or one spatial dimension. Closure is achieved by writing particle and energy fluxes in a local approximation as products of density and temperature gradients with diagonal and off-diagonal transport coefficients. ${ }^{7,8}$ In the limit of large radial electric fields treated by Kovrizhnykh, 8 these coefficients take a particularly simple form which is convenient for the numerical calculations. In this paper, we take an alternate kinetic approach in which the bounce-averaged drift kinetic equation is solved directly for the distribution function. To reduce the problem to one which is tractable with current computing capabilities, the distribution function is assumed isotropic in pitch angle and calculated as a function of radius and speed on a two-dimensional finite difference mesh. This nonstandard approach has an advantage over moment methods in that it retains the implicit coupling between real and velocity space, and no functional forms for local diffusion coefficients such as those of Kovrizhnykh need be assumed. In particular, a careful accounting of regions in velocity space where electric and magnetic poloidal drifts cancel (super banana particle orbits) leads to enhanced losses which significantly modify the ambipolar potential.

In Sect. 2, we derive the three simultaneous equations used to describe the evolution of an isotropic particle distribution in a large aspect rat1o EBT toroidal plasma. Integration over velocity shows that particles and energy are conserved. A simple analytic solution to these equations in Sect. 3 assumes a nonconservative form of the Krook ${ }^{9}$ collision operator and an unperturbed distribution function which is Maxwellian. In the large radial electric field limit, this yields a result similar to that of Kovrizhnykh. ${ }^{8}$ In Sect. 4, we develop a full Fokker-Planck representation of the collision operator including Coulomb, microwave, 1onizing, and 
charge-exchange collisions. Numerical results using this operator are discussed in detail in Sect. 5. Stable, radially inward pointing ambipolar electric fields appear to exist as a result of enhanced loss regions near the center of the machine where ion poloidal drifts cancel.

\section{THE DRIFT KINETIC EQUATION FOR EBT}

We begin with the bounce-averaged drift kinetic equation ${ }^{6}$ for the distribution of guiding centers $f=f(r, \theta, v, \alpha, t)$,

$\frac{\partial f}{\partial t}+\vec{v}_{D} \cdot \nabla f+\frac{e}{m} \frac{\vec{v}_{D}}{v} \cdot \vec{E} \frac{\partial f}{\partial v}+\frac{\tan \alpha}{2} \vec{v}_{D} \cdot\left(\frac{\nabla B}{B}-\frac{2 e \vec{E}}{m v^{2}}\right) \frac{\partial f}{\partial \alpha}=C(f)$,

where $v$ is the speed, $\vec{v}_{D}$ is the bounce-averaged guiding center drift velocity, $C(f)$ is the collision integral, $\vec{E}$ is the electric field, $m$ and $e$ are the particle mass and charge respectively, and $\alpha=\tan ^{-1}\left(v_{1} / v_{\|}\right)$is the pitch angle where $v_{1}$ and $v_{\|}$are the components of velocity perpendicular and parallel to the magnetic field $B$. Choosing toroidal coordinates $r$ and $\theta$ as in Fig. 1, $\vec{v}_{D}$ may be written,

$\overrightarrow{\mathrm{v}}_{\mathrm{D}}=\left(\mathrm{v}_{\mathrm{Dy}} \sin \theta\right) \hat{\mathrm{r}}+\left(\mathrm{r} \Omega+\mathrm{v}_{\mathrm{Dy}} \cos \theta\right) \hat{\theta}$,

where $\Omega$ is the poloidal drift frequency and $v_{\text {ny }}$ is the vertical drift velocley due to toroidal curvature. We assume a purely radial electric field, $E=E_{r}$, and a purely axial magnetic field, $B=B_{z}=B_{0} / h$, where $h=$. $1+r / R_{T} \cos \theta$ and $\dot{R}_{T}$ is the majur radius of the torus. The poloidal drift frequency may then he writton $3 \mathrm{c}$,

$\Omega=\Omega_{0}-\frac{E}{B_{0} R_{T}} \cos \theta$,

where

$\Omega_{0}=\Omega_{\nabla B}-\frac{E}{r_{0}}$

The poloidal drift frequency $\Omega_{\nabla B}$ is due to mirrorlike gradients and curvature in the magnetic field. 
ORNL-DWG 77-12491

TOROIDAL COORDINATE SYSTEM

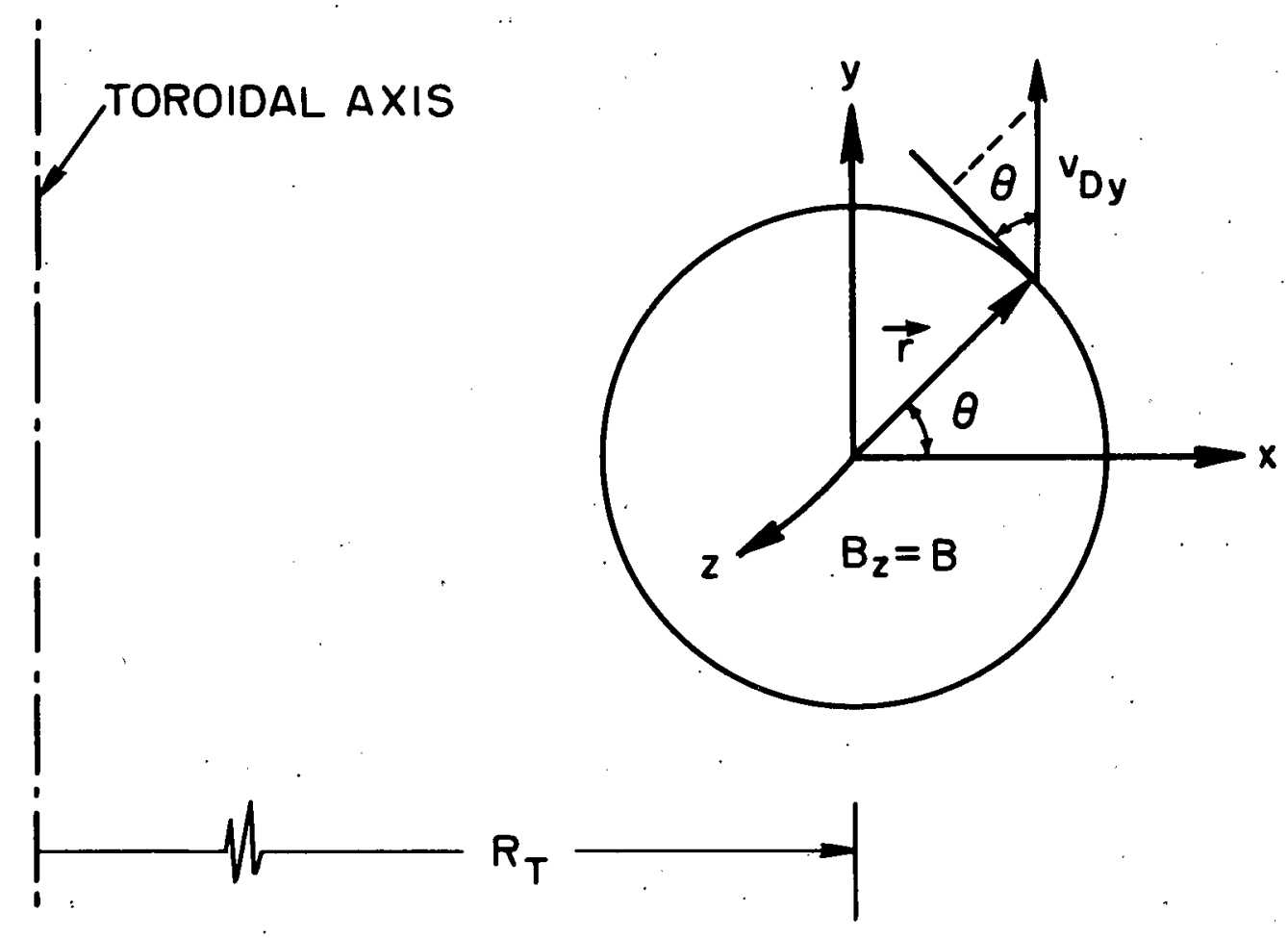

Fig. 1. Toroidal coordinate system. 
To simplify Eq. (1) and reduce the problem to one which can be treated with standard two-dimensional finite difference techniques, we assume $f$ to be isotropic in pitch angle thereby eliminating the last term on the left side of Eq. (1). Likewise we write the poloidal and vertical drifts as

$v_{D y}=\frac{-m v^{2}}{3 e B_{0} R_{T}}$,

$\Omega_{V B}=\frac{-m v^{2}}{3 e B R_{o} r}$,

where $K_{c}$ is the average radius of curvature for $\mathrm{N}_{s}$ mirror sectors which we approximate as $^{7}$

$\frac{1}{R_{c} r}=\frac{2}{R_{T}^{2}}\left(\frac{N_{s}}{2}\right)^{2}$.

With these assumptions, Eq. (1) simplifies to

$$
\begin{array}{r}
\frac{\partial f}{\partial t}+v_{D y} \sin \theta \frac{\partial f}{\partial r}+\left(\Omega_{0}-\frac{E}{B_{0} R_{T}} \cos \theta+\frac{v_{D y}}{r} \cos \theta\right) \frac{\partial f}{\partial \theta}+\frac{e E}{m} \frac{v_{D y}}{v} \sin \theta \frac{\partial f}{\partial v} \\
=C(f) .
\end{array}
$$

We use a Fokker-Planck representation of the collision operator

$$
C(f)=a(f) \frac{\partial^{2} f}{\partial v^{2}}+b(f) \frac{\partial f}{\partial v}+c(f) f+d(f)
$$

and assume that the coefficients $a, b, c$, and $d$ are linear functions of $f$. Particular expressions for these coefficients depend on the collision process considered and are derived in the next section.

To further simplify the problem, we Fourier expand the distribution function in the poloidal angle $\theta$,

$f=f_{o}+f_{c} \cos \theta+f_{s} \sin \theta+\ldots$. 
Keeping lowest order terms in the expansion, Eq. (8) can be written

$$
\begin{array}{r}
c(f)=\left\{C_{0}\left(f_{0}\right)+\frac{1}{2}\left[C_{c}\left(f_{c}\right)+C_{s}\left(f_{s}\right)\right]\right\}+\cos \theta\left[C_{0}\left(f_{c}\right)+C_{c}\left(f_{0}\right)\right]+\sin \theta \\
\times\left[c_{0}\left(f_{s}\right)+C_{s}\left(f_{o}\right)\right],
\end{array}
$$

where we have used the notation

$c_{a}\left(f_{b}\right)=a\left(f_{a}\right) \frac{\partial^{2} f_{b}}{\partial v^{2}}+b\left(f_{a}\right) \frac{\partial f_{b}}{\partial v}+c\left(f_{a}\right) f_{b}+d\left(f_{a}\right)$

Substituting Eqs. (9) and (10) into Eq. (7), keeping lowest order terms in the poloidal expansion, and equating powers of $\sin \theta$ and $\cos \theta$, we arrive at the final set of three simultaneous equations solved in the model

$$
\begin{array}{r}
\frac{\partial f_{o}}{\partial t}+\frac{1}{2}\left[v_{D y}\left(\frac{\partial f_{s}}{\partial r}+\frac{f_{s}}{r}\right)-\frac{E f}{B_{o} R_{T}}\right]+\frac{1}{2} \frac{e E}{m v} v_{D y} \frac{\partial f_{s}}{\partial v}=C_{o}\left(f_{o}\right) \\
+\frac{1}{2}\left[C_{c}\left(f_{c}\right)+C_{s}\left(f_{s}\right)\right]
\end{array}
$$

$$
\begin{aligned}
& \frac{\partial f_{c}}{\partial t}+\Omega_{o} f_{s}=C_{o}\left(f_{c}\right)+C_{c}\left(f_{o}\right), \\
& \frac{\partial f}{\partial t}-\Omega_{o} f_{c}+v_{D y} \frac{\partial f}{\partial r}+\frac{e E}{m v} v_{D y} \frac{\partial f}{\partial v}=C_{o}\left(f_{s}\right)+C_{s}\left(f_{o}\right) .
\end{aligned}
$$

We check to see that the kinetic model conserves particles by integrating Eq. (12a) over velocity and averaging over $\theta$. We obtain the continuity equation,

$$
\frac{\partial n}{\partial t}+\frac{1}{r} \frac{\partial}{\partial r}\left(r \Gamma_{r}\right)=\nu_{i} n
$$

where $\nu_{i}$ is the ionization rate, and the particle density $n$ and radial particle flux $\Gamma_{r}$ are defined as 


$$
\begin{aligned}
& n=\int \frac{h d \theta}{2 \pi} \int f^{3} \vec{v}=4 \pi \int_{0}^{\infty} v^{2} f_{0} d v, \\
& \Gamma_{r}=\int \frac{h d \theta}{2 \pi} \int f v_{D r} d^{3 \vec{v}}=4 \pi \int_{0}^{\infty} v^{2} \frac{f_{s} v_{D y}}{2} d v .
\end{aligned}
$$

Similarly, conservation of energy is obtained by multiplying Eq. (12a) by $1 / 2 \mathrm{mv}^{2}$ before taking the velocity integral. Averaging over $\theta$ then yields the energy equation

$\frac{\partial}{\partial t}\left(\frac{3}{2} n k T\right)+\frac{1}{r} \frac{\partial}{\partial r}\left(r \eta_{r}\right)=a E \Gamma r=\int \frac{h d \theta}{2 \pi} \int C_{0}\left(f_{0}\right) \frac{m v^{2}}{2} d^{3} \vec{v}$,

where the energy density and radial heat flux are defined respectively as

$$
\begin{aligned}
& \frac{3}{2} \mathrm{nkT}=\int \frac{\mathrm{hd} \theta}{2 \pi} \int \frac{1}{2} \mathrm{mv}^{2} \mathrm{fd}^{3} \overrightarrow{\mathrm{v}}=2 \pi \mathrm{m} \int_{0}^{\infty} \mathrm{v}^{4} \mathrm{f}_{\mathrm{o}} \mathrm{dv} \\
& \mathrm{Q}_{\mathrm{r}}=\int \frac{\mathrm{hd} \theta}{2 \pi} \int \frac{1}{2} \mathrm{mv}^{2} \mathrm{fv} \mathrm{Dy}_{\mathrm{D}} \mathrm{d}^{3} \overrightarrow{\mathrm{v}}=2 \pi \mathrm{m} \int_{0}^{\infty} \mathrm{v}^{4} \frac{\mathrm{f}_{\mathrm{s}} \mathrm{vy}}{2} \mathrm{dv} .
\end{aligned}
$$

In the one-dimensional or fluid transport mode $1,5 \mathrm{f}_{0}$ is assumed to be a Maxwellian distribution, and a local diffusion approximation replaces Eqs. (14b) and (16b), thus truncaling the sertes of moments and allowing a solution of Eqs. (13) and (15) for radial profiles of density $n(r)$ and temperature $T(r)$. The kinetic transport model described here, on the other hand, has the advantage that Eq. (12) is solved directly for the distribution function $f(r, v)$ without recourse to a local approximation or diffusion assumpition. Equation ( 12 ) is solved in the two-dimensional ( $r, v$ ) coordinate system so that close coupling hetween real spare and volocity space is retained. Also, the assumption of a Maxwellian distribution $f_{0}$ is not required. The disadvantage of the kinetic approach is that isotropy in velocity space must be assumed to avoid a full three-dimensional computation. 


\section{SIMPLE ANALYTIC RESULTS FOR AN UNPERTURBED DISTRIBUTION FUNCTION WHICH IS MAXWELLIAN}

A simple analytic solution to $\mathrm{Eq}$. (12) can be found in the large electric field limit for a Maxwellian distribution $\mathrm{f}_{\mathrm{o}}$ by using the nonconservative form of the Krook collision operator ${ }^{9}$

$C(f) \approx v\left(f_{0}-f\right)=-v f_{c} \cos \theta-v f_{s} \sin \theta$

where $\nu$ is the Coulomb collision frequency assumed independent of velocity (constant mean free time assumption) and

$f_{0}=\frac{n}{\pi^{3 / 2} \alpha^{3}} e^{-v^{2} / \alpha^{2}}$

with $\alpha^{2}=2 \mathrm{kT} / \mathrm{m}$. The steady-state form of $\mathrm{Eq}$. (12) can then be solved for $\mathrm{f}_{\mathrm{s}}$ as

$f_{s}=\frac{-v v_{D y}}{v^{2}+\Omega_{o}^{2}}\left(\frac{\partial f_{o}}{\partial r}+\frac{e E}{m v} \frac{\partial f_{o}}{\partial v}\right)$.

Substituting Eqs. (5), (18), and (19) into Eqs. (14b) and (16b) yields in the large field Iimit $\left(e E R_{c} \gg k T\right)$,

$\Gamma_{r}=\frac{5}{24} \frac{v v_{0}^{2}}{\nu^{2}+\Omega_{0}^{2}}\left(-\frac{\partial n}{\partial r}-\frac{2 n}{T} \frac{\partial T}{\partial r}+\frac{e E n}{k T}\right)$,

$Q_{r}=\frac{7}{2} k T \frac{5}{24} \frac{v v_{o}^{2}}{v^{2}+\Omega_{o}^{2}}\left(-\frac{\partial n}{\partial r}-\frac{3 n}{T} \frac{\partial T}{\partial r}+\frac{e E n}{k T}\right)$,

where $v_{0}=2 \mathrm{kT} /\left(e B_{0} R_{T}\right)$. Equation (20) gives a particle flux five times larger than that computed by Kovrizhnykh ${ }^{8}$ in the large field limit using the full Landau collision operator and two dimensions in velocity space. 


\section{THE COLLISION OPERATOR}

\subsection{Coulomb Collisions}

We use the Fokker-Planck representation of the Coulomb collision operator $C_{v}(f)$ for isotropic distributions. 10 For the $k^{\text {th }}$ species

$c_{v}\left(f_{k}\right)=a_{v}\left(f_{k}\right) \frac{\partial^{2} f_{k}}{\partial v^{2}}+b_{v}\left(f_{k}\right) \frac{\partial f_{k}}{\partial v}+c_{v}\left(f_{k}\right) f_{k}$,

where for $v \neq 0$,

$a_{v}\left(f_{k}\right)=\pi^{3 / 2} \gamma_{k} \sum_{j}\left(\frac{1}{3} \int_{0}^{v} d v^{\prime} \frac{v^{\prime 4}}{v^{3}} E_{j}+\frac{1}{3} \int_{v}^{\infty} d v^{\prime} v^{\prime} r_{j}\right)$,

$b_{v}\left(f_{k}\right)=\pi^{3 / 2} \gamma_{k} \sum_{j}\left[\int_{0}^{v} d v^{\prime} f_{j}\left(\frac{m_{k}}{m_{j}}-\frac{v^{\prime 2}}{3 v^{2}}\right) \frac{v^{\prime 2}}{v^{2}}+\frac{2}{3} \int_{v}^{\infty} d v^{\prime} \frac{v^{\prime}}{v} f_{j}\right]$,

$c_{v}\left(f_{k}\right)=\pi^{3 / 2} \gamma_{k} \sum_{j} \frac{m_{k}}{m_{j}} f_{j}$,

with $\gamma_{k}=e_{k}^{4}$ In $\Lambda /\left(\pi^{3 / 2} \varepsilon_{o}^{2} m_{k}^{2}\right)$ and $\Lambda=12 \pi\left(\varepsilon_{o} k T / e^{2}\right)^{3 / 2} / n^{1 / 2}$. In the limit as $\mathrm{v} \rightarrow 0$, Eq. (22) may still be used when the coefflclents are defined as

$$
\begin{aligned}
& a_{v}\left(f_{k}\right)=\pi^{3 / 2} \gamma_{k} \sum_{j} \int_{v}^{\infty} v^{\prime} d v^{\prime} f_{j}, \\
& b_{v}\left(f_{k}\right)=0, \\
& c_{v}\left(f_{k}\right)=11^{3 / 2} \gamma_{k} \sum_{j} m_{j}^{m_{k}} I_{j} .
\end{aligned}
$$

In the case of a Maxwellian distribution as defined in Eq. (18), the integrals may be expressed in terms of error integrals erf $(u)=$ $(2 / \sqrt{\pi}) \int_{0}^{u} e^{-u^{2}} d u$ giving for $v \neq 0$, 


$$
\begin{aligned}
& a_{v}\left(f_{o k}\right)=\frac{n \gamma_{k}}{4 v} \sum_{j} \frac{1}{u_{j}^{2}}\left[\frac{\sqrt{\pi}}{2} \operatorname{erf}\left(u_{j}\right)-u_{j} e^{-u_{j}^{2}}\right], \\
& b_{v}\left(f_{o k}\right)=\frac{n \gamma_{k}}{2 v^{2}} \sum_{j}\left\{\left(\frac{m_{k}}{m_{j}}-\frac{1}{2 u_{j}^{2}}\right)\left[\frac{\sqrt{\pi}}{2} \operatorname{erf}\left(u_{j}\right)-u_{j} e^{-u_{j}^{2}}\right]+u_{j} e^{-u_{j}^{2}}\right\}, \\
& c_{v}\left(f_{o k}\right)=n \gamma_{k} \sum_{j} \frac{m_{k}}{m_{j}} \frac{e^{-u_{j}^{2}}}{\alpha_{j}^{3}},
\end{aligned}
$$

and in the limit as $\dot{v} \rightarrow 0$

$$
\begin{aligned}
& a_{v}\left(f_{o k}\right)=n \gamma_{k} \sum_{j} \frac{1}{2 \alpha_{j}^{3}}, \\
& b_{v}\left(f_{o k}\right)=0, \\
& c_{v}\left(f_{o k}\right)=n \gamma_{k} \sum_{j} \frac{m_{k}}{m_{j}} \frac{1}{\alpha_{j}^{3}} .
\end{aligned}
$$

Substituting Eqs. (25), (26), and (18) into Eq. (22), it is easy to show that $C_{v o}\left(f_{0}\right)=0$ for a single species or for two species with equal temperatures. Likewise, integrating Eq. (22) over velocity, we can show that the Coulomb collision operator. conserves both particles and energy, i.e.,

$$
\begin{aligned}
& \int c_{v}\left(f_{k}\right) d^{3} \vec{v}=0, \\
& \int c_{v}\left(f_{k}\right) \frac{m}{2} v^{2} d^{3} \vec{v}=0 .
\end{aligned}
$$

\subsection{Ionization and Charge-Exchange Collisions}

The collision operator $C(f)$ includes processes other than simple Coulomb collisions treated in Sect. 4.1, for example ionizing and chargeexchange collisions which we denote by $C_{i}(f)$ and $C_{c x}(f)$, respectively.

We assume that particles created by ionization are born as a Maxwellian velocity distribution with the temperature of the neutrals, $\mathrm{T}_{0}$, that is 
$C_{i}(f)=\nu_{i} \frac{n}{\pi 3 / 2 \alpha_{0}^{3}} e^{-v^{2} / \alpha^{2}}$,

where $\alpha_{0}^{2}=2 \mathrm{kT}_{0} / \mathrm{m}$ and $\nu_{i}$ is the ionization rate, $n_{0}\langle\sigma v\rangle_{i}$, where $n_{0}$ is the neutral density and $\left\langle\sigma v{ }_{i}\right.$ is the cross section for ionization. Integrating Eq. (28) over velocity yields

$\int C_{i}(f) d^{3} \vec{v}=v_{i} n$

$\int C_{i}(r) \stackrel{m}{2} v^{2} d^{3} \vec{v}=v_{i}\left(\frac{3}{2} n k T_{0}\right)$

Therefore, ionizing collisions conserve neither particles nor energy. The result in Eq. (29a) has been used explicitly in writing Eq. (13).

Likewise, we assume that charge-exchange ions are born as a Maxwellian velocity distribution with the temperature of the neutrals

$c_{c x}(f)=-v_{c x}\left(f-\frac{n}{\pi 3 / 2 \alpha_{0}^{3}} e^{-v^{2} / \alpha_{0}^{2}}\right)$,

where $v_{c x}$ is the charge-exchange rate $n_{0}^{\langle\sigma v\rangle}{ }_{c x}$. Integrating Eq. (30) over velocity yields

$\int C_{c x}(f) d^{3} \vec{v}=0$

$\int C_{c x}(f) \frac{m}{2} v^{2} d^{3} \vec{v}^{*}=-v_{c x} \frac{3}{2} n k\left(T_{i}-T_{u}\right)$

in other words, Eq. (30) conserves the number of particles but not their energy. Energy is lost by the charge-exchange operator at the rate $v_{c x} 3 / 2 n k\left(T_{i}-T_{0}\right) W / m^{3}$. 


\subsection{Microwave Collisions}

We include the effects of electron cyclotron resonance with the microwave field in the collision operator, $C_{\mu}(f)$, which models quasi-linear diffusion in velocity spacell

$C_{\mu}(f)=\nabla_{v} \cdot\left(D_{\mu} \nabla_{v} f\right)$

where $\mathrm{D}_{\mu}$ is an effective velocity diffusion rate due to microwaves. When written in spherical coordinates in velocity space, Eq. (31) takes a form similar to the Fokker-Planck representation for Coulomb collisions in Eq. (22)

$c_{\mu}(f)=a_{\mu}(f) \frac{\partial^{2} f}{\partial v^{2}}+b_{\mu}(f) \frac{\partial f}{\partial v}+c_{\mu}^{\prime}(f) f$,

where for $v \neq 0$

$a_{\mu}(f)=D_{\mu}$,

$b_{\mu}(f)=\frac{\partial D_{\mu}}{\partial v}+2 \frac{D_{\mu}}{v}$,

$c_{\mu}(f)=0$.

In the limit $v \rightarrow 0$, we keep the form of Eq. (32) and redefine

$a_{\mu}(f)=3 D_{\mu}$,

$b_{\mu}(f)=0$,

$c_{\mu}(f)=0$.

Integrating Eq. (31) over velocity in spherical coordinates gives

$\int C_{\mu}(f) d \cdot \vec{v}=0$

$\int C_{\mu}(f) \frac{m_{e}}{2} v^{2} d^{3} \vec{v}=3 m_{e} D_{\mu}$, 
where $\mathrm{Eq}$. (35b) assumes $\mathrm{D}_{\mu}$ independent of velocity. Therefore, the microwave operator in Eq. (31) conserves particles and provides a power density input to the electrons of $\left(3 \mathrm{~m}_{\mathrm{e}} \mathrm{nD} \mathrm{D}_{\mu}\right) \mathrm{W} / \mathrm{m}^{3}$.

We choose an effective velocity space diffusion rate $D_{\mu}=0.25 \mathrm{~d}\left\langle v_{1}^{2}\right\rangle /$ dt as given by Eldridge ${ }^{12}$ where

$\frac{\mathrm{d}\left\langle\mathrm{v}_{\perp}^{2}\right\rangle}{\mathrm{dt}}=2 \pi\left(\frac{\mathrm{e}}{\mathrm{m}}\right)^{2} \sum_{\text {modes }}\left|\mathrm{E}_{\perp}\right|^{2} \sum_{\mathrm{n}=-\infty}^{\infty} \mathrm{J}_{\mathrm{n}-1}^{2}\left(\frac{\mathrm{k}_{\perp} \mathrm{v}_{1}}{\Omega}\right) \delta\left(\omega-\mathrm{n} \Omega-\mathrm{k}_{\|} \mathrm{v}_{\|}\right)$,

and $E_{\perp}$ is the complex microwave electric field amplitude perpendicular to $B, \Omega$ is the electron cyclotron frequency, and $\omega$ and $k$ are the microwave frequency and wave number, respectively. Averaging Eq. (35) over a fleld line using $\left|\mathrm{E}_{\perp}\right|^{2} \approx 2 / 3|E|^{2}$, assuming $\mathrm{v}_{\perp} \gg \mathrm{v}_{\|}$and one mode with $\mathrm{k}_{1} \approx \omega / \mathrm{c}$, $k_{\|} \approx 0$ we find

$\mathrm{D}_{\mu}=\mathrm{b}_{1} \mathrm{~J}_{\mathrm{o}}^{2}\left(\frac{\mathrm{v}}{\mathrm{c}}\right)+\mathrm{b}_{2} \mathrm{~J}_{1}^{2}\left(\frac{2 \mathrm{v}}{\mathrm{c}}\right)+\ldots$.

with

$\mathrm{b}_{1}=\frac{2 \pi}{3}\left(\left|\frac{\mathrm{eE}}{\mathrm{m}}\right|^{2} \frac{\mathrm{n}(\mathrm{s}) / \overline{\mathrm{n}}}{\mathrm{Ld} \Omega / \mathrm{ds}}\right)_{\omega=\Omega}$,

$\mathrm{b}_{2}=\frac{1}{2} \frac{2 \pi}{3}\left(\left|\frac{\mathrm{e} F}{\mathrm{~m}}\right|^{2} \frac{\mathrm{n}(\mathrm{s}) / \overline{\mathrm{n}}}{\mathrm{Ld} \Omega / \mathrm{ds}}\right)_{\omega=2 \Omega}$,

where $\bar{n}$ is the field averaged plasma density and $L$ is the separation in $z$ between resonance $z$ ones. The quantity in parentheses is to be evaluated at the position s along the field line such that $\omega=\Omega(s), 2 \Omega(s)$, etc. Assuming a spatial profile for the microwave field amplitude, the magnitude $|E|$ is estimated by integrating the power density, $3 \mathrm{~m}_{\mathrm{e}} \mathrm{nD}$, over the plasma volume and equating the result to the total power deposited in the toroidal plasma as estimated experimentally. The derivative, $d \Omega / d s$, is found by adopting a model bumpy cylinder magnetic field $B_{0}$ and applying the toroidal correction $B=B_{0} / h$. To prevent $D_{\mu}$ from becoming zero when $b_{2}=0$ and $\mathrm{J}_{0}(\mathrm{v} / \mathrm{c})=0$, we add a small term to Eq. (37) of the form $\delta b_{1} /\left(1+v^{2} / c^{2}\right)$ where $\delta \approx 10^{-3}$. 
In the nonrelativistic limit, $v / c \ll 1$, Eq. (37) reduces to the simple result of $\operatorname{Ref} .12, D_{\mu} \approx b_{1}$ which is independent of velocity.

\section{RESULTS FOR AN UNPERTURBED DISTRIBUTION FUNCTION WHICH IS MAXWELLIAN}

\subsection{Numerical Methods}

The finite difference form of Eq. (12) has been written using the collision operators developed in the previous section. The resulting set of three coupled finite difference equations has been set up for numerical solution on a grid of 20 radial points and 40 velocity points. We use an alternating difference implicit (ADI) scheme due to Douglas and Rachford 3 which is accurate to second order in the time step $\Delta t$ and requires only inversion of a tridiagonal matrix for one equation or a nine-diagonal matrix for three coupled equations as in Eq. (12). The Douglas-Rachford version of ADI advances each time interval in two steps. First, a predictor step differences the radial dimension implicitly and the velocity dimension explicitly. Then the corrector step differences the velocity dimension implicitly and the radial dimension explicitly. We find empirically that a time step $\Delta t \leqslant 8 \times 10^{-4} \mathrm{sec}$ is required for numerical stability of Eq. (12b) and (12c) while $\Delta t \leqslant 8 \times 10^{-6} \mathrm{sec}$ is needed for stability of Eq. (12a). Therefore, the unperturbed distribution $f_{0}$ adjusts rapidly with respect to the neoclassical perturbations $f_{c}$ and $f_{s}$. As an initial step in the solution of this multiple time scale problem, we calculate $f_{c}$ and $f_{s}$ for a Maxwellian distribution $f_{0}$ with fixed density and temperature profiles. In principle, at the end of the neoclassical calculation, $f_{0}$ can be readjusted to insure densities and temperatures defined by Eqs. (14a) and (16a) which are consistent with $f_{c}$ and $f_{s}$ and the neoclassical fluxes in Eqs. (14b) and (16b).

Because the bulk of the particles exist at low velocities, steep gradients in both the perturbed and unperturbed distribution function in this region require a smaller mesh spacing than at large velocities. Therefore we transform to a new velocity variable $w=1 / m_{s}$ ln $\left(1+m_{s} v\right)$ such that $\mathrm{dv} / \mathrm{dw}=1+\ddot{m}_{\mathrm{s}} \mathrm{v}$ is a lincarly increasing function of $v$ with slope 
$\mathrm{m}_{\mathrm{s}}$. The radial coordinate is left unchanged. This allows a solution of the problem on a uniform mesh in w which corresponds to a linearly increasing mesh in the real velocity variable $v$.

\subsection{Neoclassical Fluxes and Diffusion Rates}

We assume typical parameters corresponding to EBT I with $\mathrm{N}_{S}=24$ mirror sectors and a major radius $R_{T}=150 \mathrm{~cm}$. The plasma radius is taken as $a=10 \mathrm{~cm}$ with a resonant magnetic field, $B_{0}=6.5 \mathrm{kG}$. The unperturbed discribueion $\tilde{f}_{0}$ is taken to be Maxwellian with zero temperature gradient and a parabolic density profile

$n(r)=\left(n_{1}-n_{2}\right)\left(1-\frac{r^{2}}{a^{2}}\right)+n_{2}$,

with central density $\mathrm{n}_{1}=2 \times 10^{12} \mathrm{~cm}^{-3}$ at $\mathrm{r}=0$ and edge density $\mathrm{n}_{2}=$ $3 \times 10^{11} \mathrm{~cm}^{-3}$ at $\mathrm{r}=\mathrm{a}$. Ion and electron temperatures are chosen as $\mathrm{kT}_{i}=$ $36 \mathrm{eV}$ and $\mathrm{kT}_{\mathrm{e}}=140 \mathrm{eV}$, respectively. With these assumptions and an ad hoc electric potential profile

$\phi=\phi_{0}\left(1-\frac{r^{2}}{a^{2}}\right)$

Eqs. (12l) alld (12c) are solved numerically for $f_{c}$ and $f_{s}$ as functions of $r$ and $w$. Typical results are plotted in Fig. 2 for ions with $\phi_{0}=-50 \mathrm{v}$. Resulting particle fluxes, $\Gamma_{r}$, computed from $E q .(14 b)$ and evaluated al $r / a=0.5$, are plotted in $F i g .3$ as functions of $\phi_{0}$. Positive and ncgative fluxes are represented by solid and dashed curves respertively and aro shown for both ions $\left(\Gamma_{i}\right)$ and electrons $\left(\Gamma_{e}\right)$. We note that for positive electric tields $\left(\phi_{0}>0\right)$ the electron flux reverses sign for potentials exceeding the electron thermal energy, $\phi_{0}>\mathrm{kT}_{\mathrm{e}} /|\mathrm{e}|$, and for negative fields $\left(\phi_{0}<0\right)$ the ion flux reverses sign for potentials less than the ion thermal energy, $\phi_{0}<-\mathrm{kT}_{i} /|\mathrm{e}|$. Negative fluxes occur only because of the ad hoc electric field assumption. In reality, a self-consistent electric field forms to electrostatically decrease the outward flux of the faster diffusing species and increase the outward flux of the slower diffusing species giving a resultant outward or positive flux which is the same for 
ORNL/DWG/FED-77926
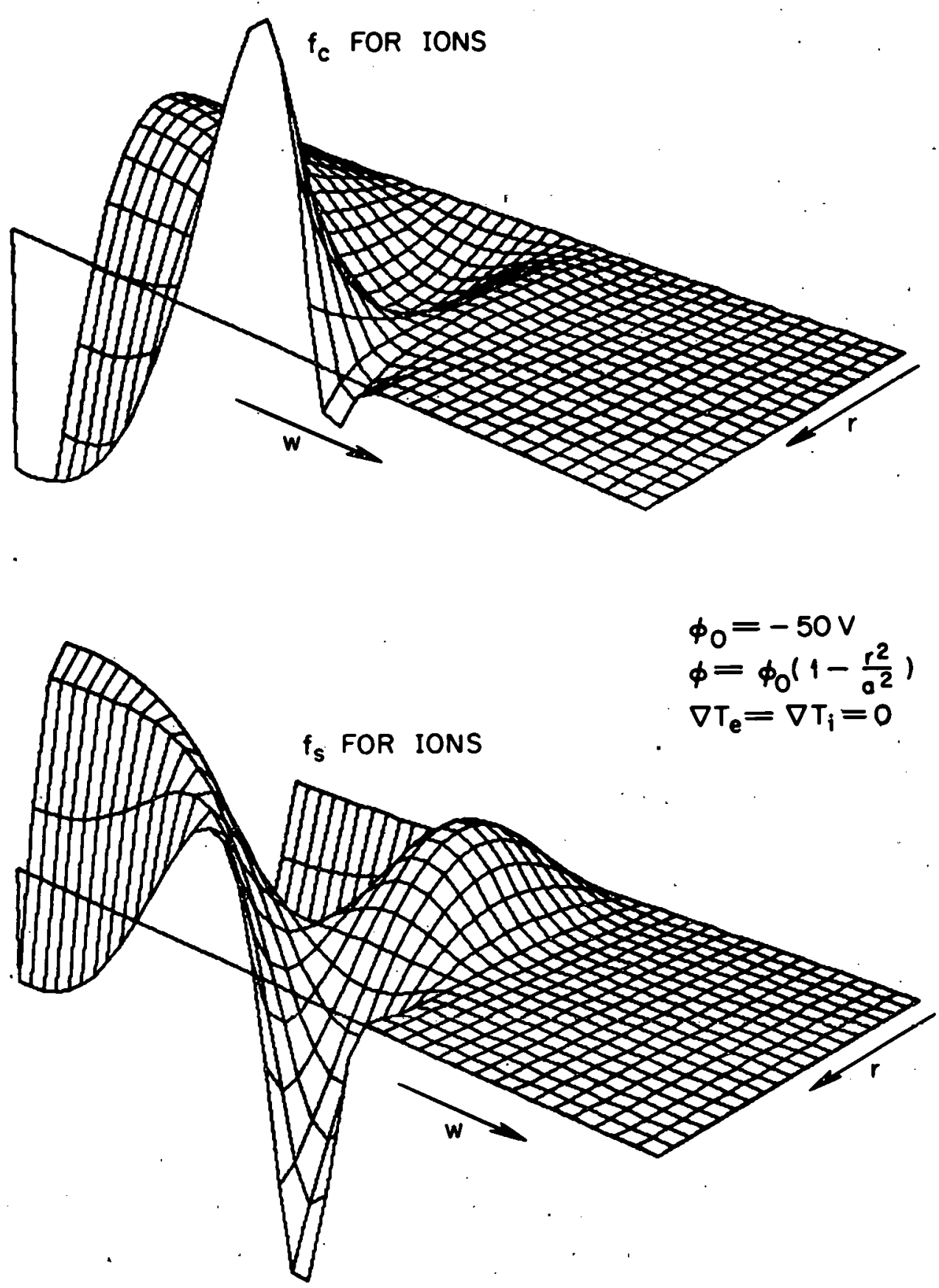

Fig. 2. Typical numerical results showing the perturbed distribution functions $f_{c}$ and $f_{s}$ for ions with $\phi_{o}=-50 \mathrm{~V}$. 
both species. The results in Fig. 3 indicate where this self-consistent potential lies for the case considered, that is in the regions near $\phi_{0}=$ $\mathrm{kT}_{\mathrm{e}} /|\mathrm{e}|$ and $\phi_{0}=-\mathrm{kT}_{i} /|\mathrm{e}|$ where the outward fluxes of ions and electrons are of comparable magnitude. This result could have been predicted in advance from the approximate solution to Eqs. (12b) and (12c) given in Eq. (20). Assuming no temperature gradients and parabolic profiles for $n(r)$ and $\phi(r)$ as in Eqs. (39) and (40), the flux given by Eq. (20) reverses sign near $\phi_{j} \approx-k T_{j} / e_{j}$ where $j=e, i$ rnrresponds to electronc and ions, respectively. This agrees closely with Fig. 3. If we assume parabolic temperatures as well as densities in Eq. (20), flux reversal occurs near. $\phi_{0} \approx$ $-3 \mathrm{kT}_{j} / \mathrm{e}_{j}$. Repeating the calculations of $\mathrm{Fig}$. 3 with temperature profiles,

$$
\begin{aligned}
& T_{e}(r)=\left(T_{e 1}-T_{e 2}\right)\left(1-\frac{r^{2}}{a^{2}}\right)+T_{e 2}, \\
& T_{i}(r)=\left(T_{i 1}-T_{i 2}\right)\left(i-\frac{r^{2}}{a^{2}}\right)+T_{i 2},
\end{aligned}
$$

where $\mathrm{T}_{\mathrm{el}}=140 \mathrm{eV}, \mathrm{T}_{\mathrm{e} 2}=40 \mathrm{eV}, \mathrm{T}_{11}=36 \mathrm{eV}$, and $\mathrm{T}_{i_{2}}=13.5 \mathrm{eV}$, we find flux reversal and hence self-consistent potentials near $\phi_{0} \approx 5 \mathrm{kT} \mathrm{e}_{\mathrm{e}} / \mathrm{e} \mid$ and $\phi_{0} \approx-5 \mathrm{kT}_{i} /|\mathrm{e}|$. These results, illustrated in Fig. 4, indicate that the simple large electric field result of Eq. (20) underestimates the effoct of particle diffusion due to temperature gradients. In reality, s.lightiy larger potentials are required to produce flux reversal than might be expected from the simple model.

of particular interest in Fig. 4 is the negative electric field solution with a self-consistent potential on the order of $-200 \mathrm{~V}$. This result is in close agreement with current experimental observations. ${ }^{2}$ A careful accounting of loss regions where electric and magnetic poloidal drifts cancel (super banana particle orbits) in the present kinetic model leads to ion loss rates which are in some cases two orders of magnitude greater than electron rates (see for example Figs. 3 and 4 for $-200 \leqslant \phi_{0} \leqslant 0$ ). In these cases, radially inward pointing self-consistent electric fields result with potentials on the order of a few times the ion temperature.

This is made clear by the solid lines in Fig. 5 where we approximate ion diffusion rates from the zero temperature gradient results of Fig. 2 


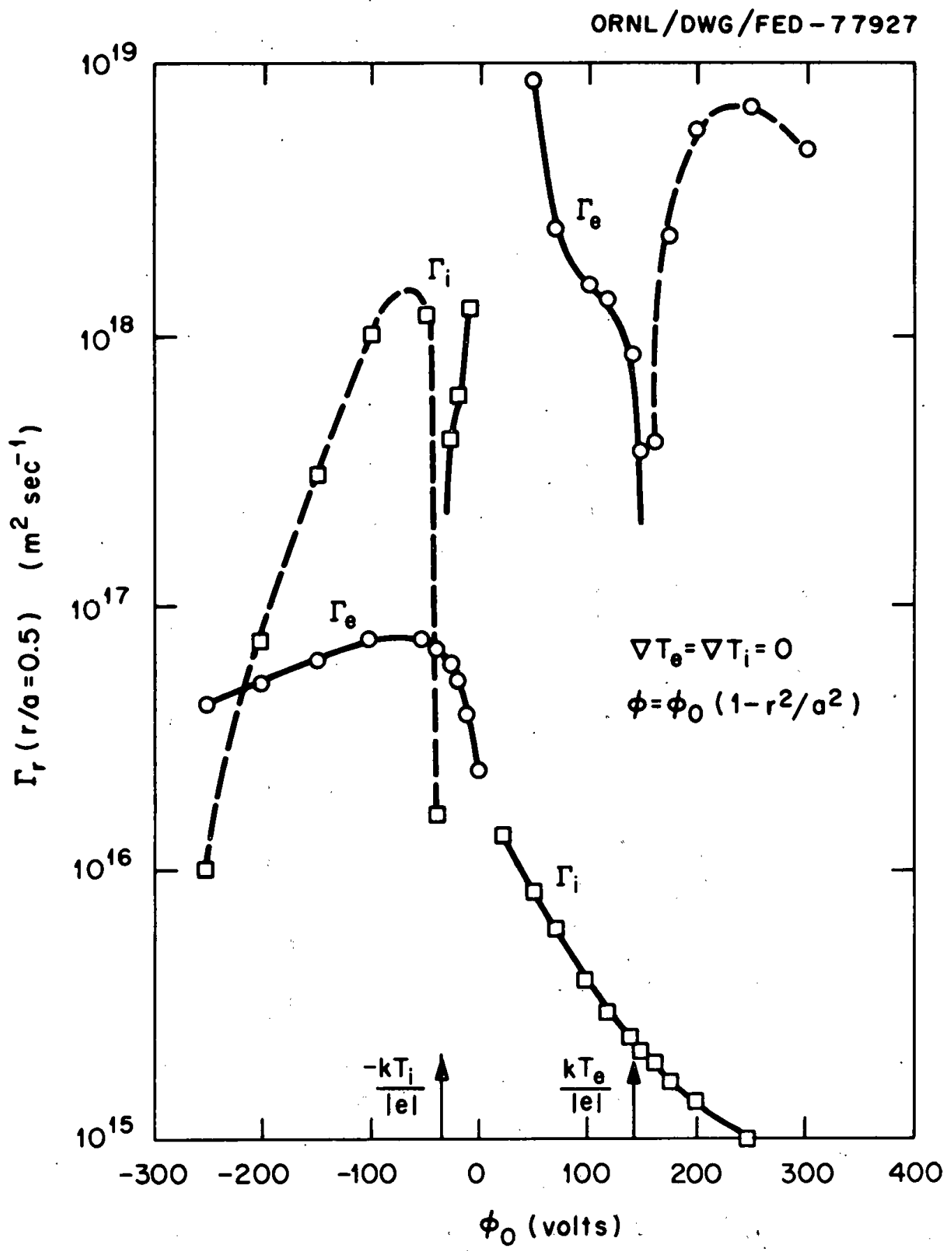

Fig. 3. Numerical results showing the dependence of particle flux on electric potential. The unperturbed distribution function is taken as a Maxwellian distribution with no temperature gradient. The electric potential profile is assumed parabolic. 


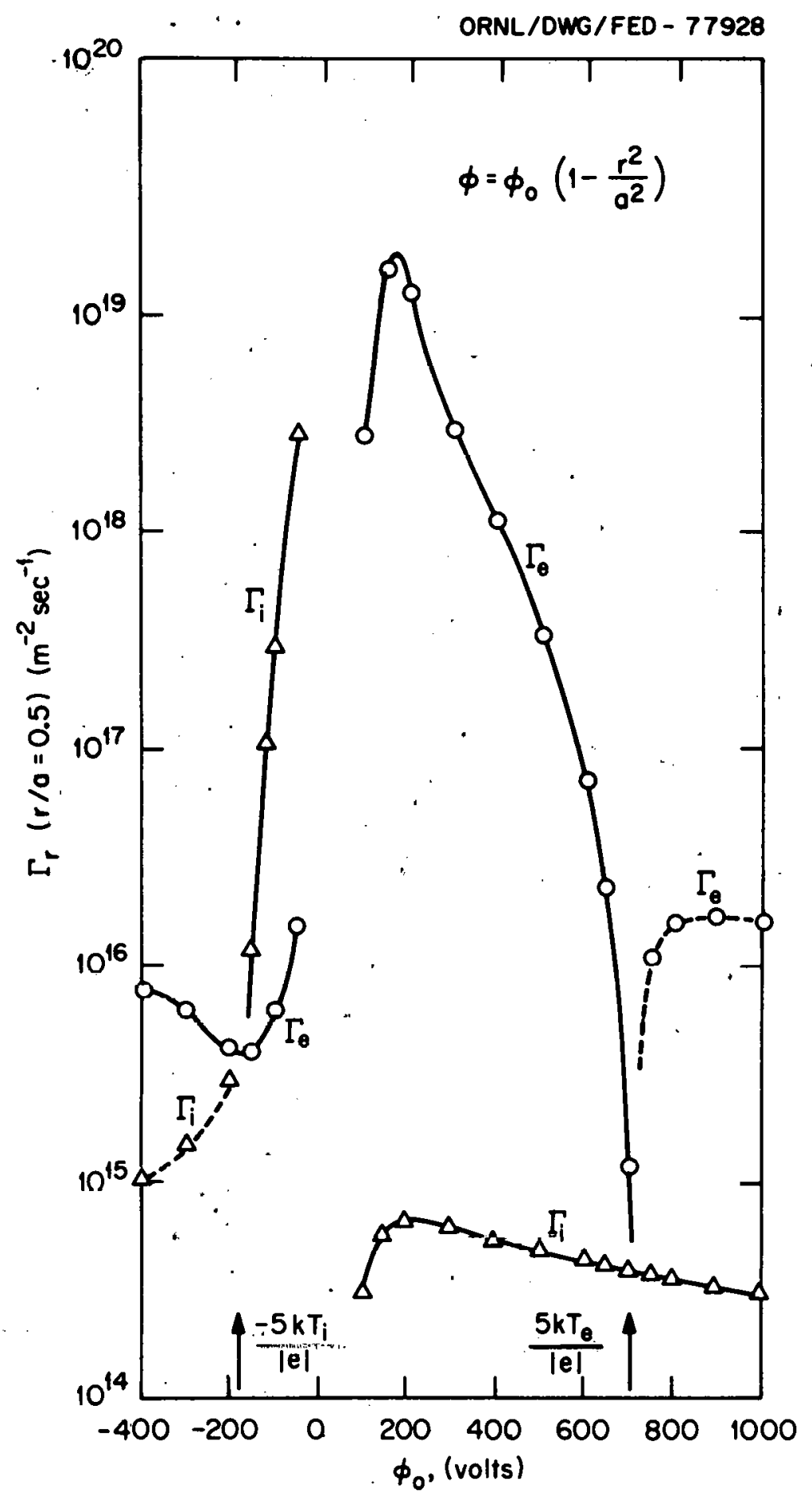

Fig. 4. Numerical results as in Fig. 2, but including nonzero temperature gradients. 


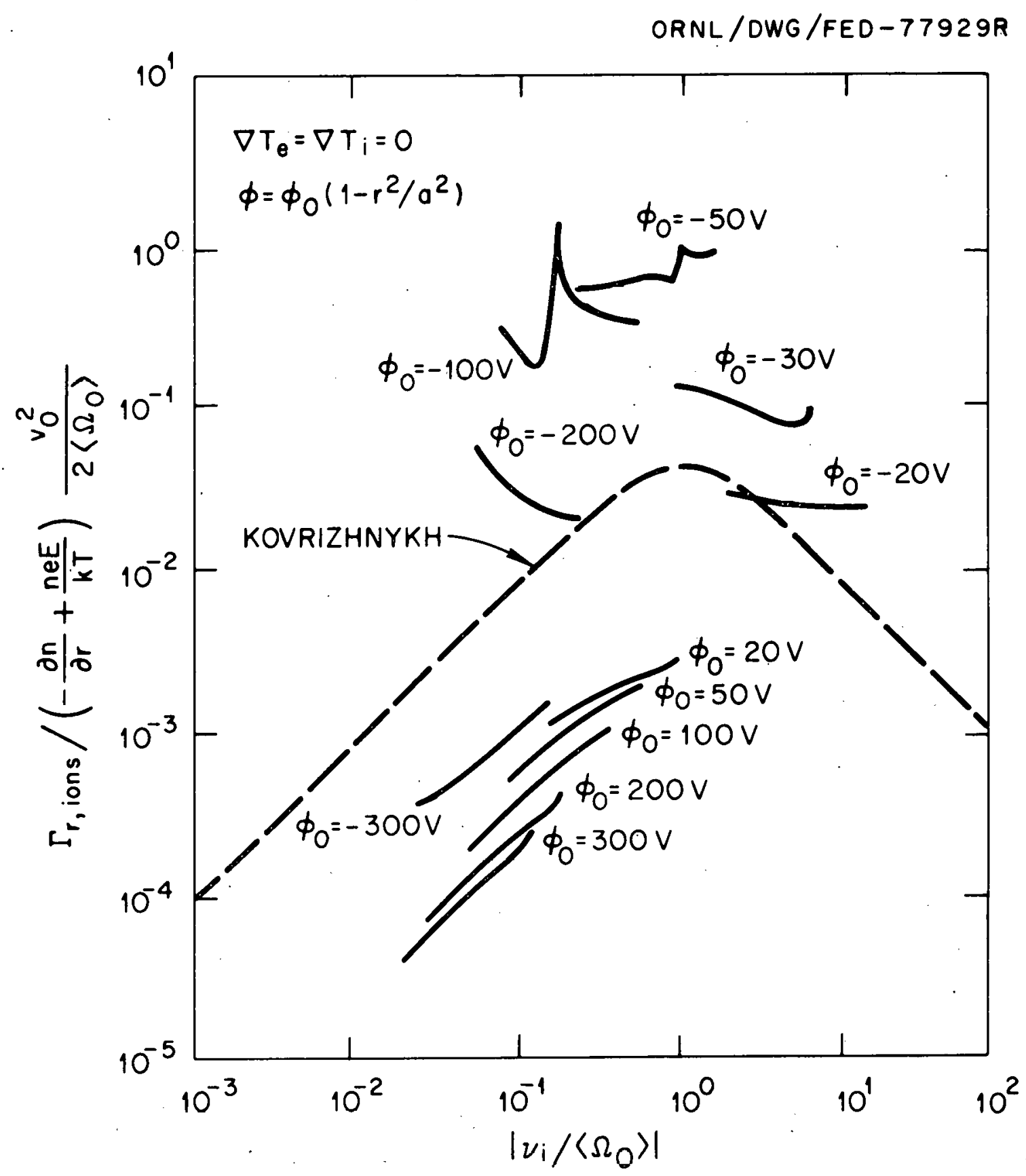

Fig. 5. Diffusion rates from the results of Fig. 2; the dashed line shows Kovrizhnykh's result. 
In the large field limit of Kovrizhnykh, ${ }^{8} \mathrm{ER}_{\mathrm{c}} \gg \mathrm{kT} / \mathrm{e}$ and these enhanced loss regions are pushed far out on the tail of the particle distribution function where they have little effect. Therefore asymmetry between positive and negative potentials does not appear in the dashed curve of Fig. 5 .

A question naturally arises as to the sensitivity of these results to the ad hoc electric potential profile chosen in Eq. (40) since the cancellation of poloidal drifts in Eq. (41) occurs uniformly in radius only for a parabolic potential profile. For a cubic profile, $\phi=\phi_{0}\left(1-r^{3} / a^{3}\right)$, Eq. (41) gives $<\Omega_{0}>=0$ only at the discrete radial positions $\frac{\mathrm{r}}{\mathrm{a}}=-\frac{2}{3}\left(\frac{\mathrm{a}}{\mathrm{R}_{\mathrm{T}}} \frac{\mathrm{Ns}}{2}\right)^{2} /\left(\frac{|\mathrm{e}|_{\mathrm{o}}}{\mathrm{k}^{\prime} \mathrm{l}_{\mathrm{i}}}\right)$.

This gives, for example, cancellation of ion poloidal drifts at $\mathrm{r} / \mathrm{a}=0.142$ for $\phi_{0}=-3 \mathrm{kT}_{i} /|\mathrm{e}|$, or at $\mathrm{r} / \mathrm{a}=0.0854$ for $\phi_{0}=-5 \mathrm{kT}_{i} /|\mathrm{e}|$. However, this spatially discrete character of the cancellation apparently does not reduce the enhancement of diffusion as shown by Fig. 6 where the cubic profile has been used with results remarkably similar to those in Fig. 4. Thus we conclude that our results are relatively insensitive to the electric potential profile used.

Another question concerns the relative stability of the two roots which appear in Figs. 4 and 6 . One-dimensional transport models 5 show that self-consistent electric fields are proportional to pressure gradients, and the resulting ambipolar diffusion rates are dominated by the slower diffusing species. Hence, the positive field root in Figs. 4 and 6 tends to be unstable in the sense that a positive perturbation in the potential $\phi_{0}$ decreases the diffusion rate of the slower species, in this case the ions. This leads to larger pressure gradients to maintain the loss rate and hence larger potentials. In one-dimensional transport models, this positive feedback on the potential can be stabilized ${ }^{5}$ by assuming a collisionless diffusion rate proportional to $(\Omega / v)^{2}$.

By contrast, the negative field root in Figs. 4 and 6 appears to be stable since the diffusion rate of the slower species, in this case the electrons, is approximately minimum where the root occurs. Thus a 


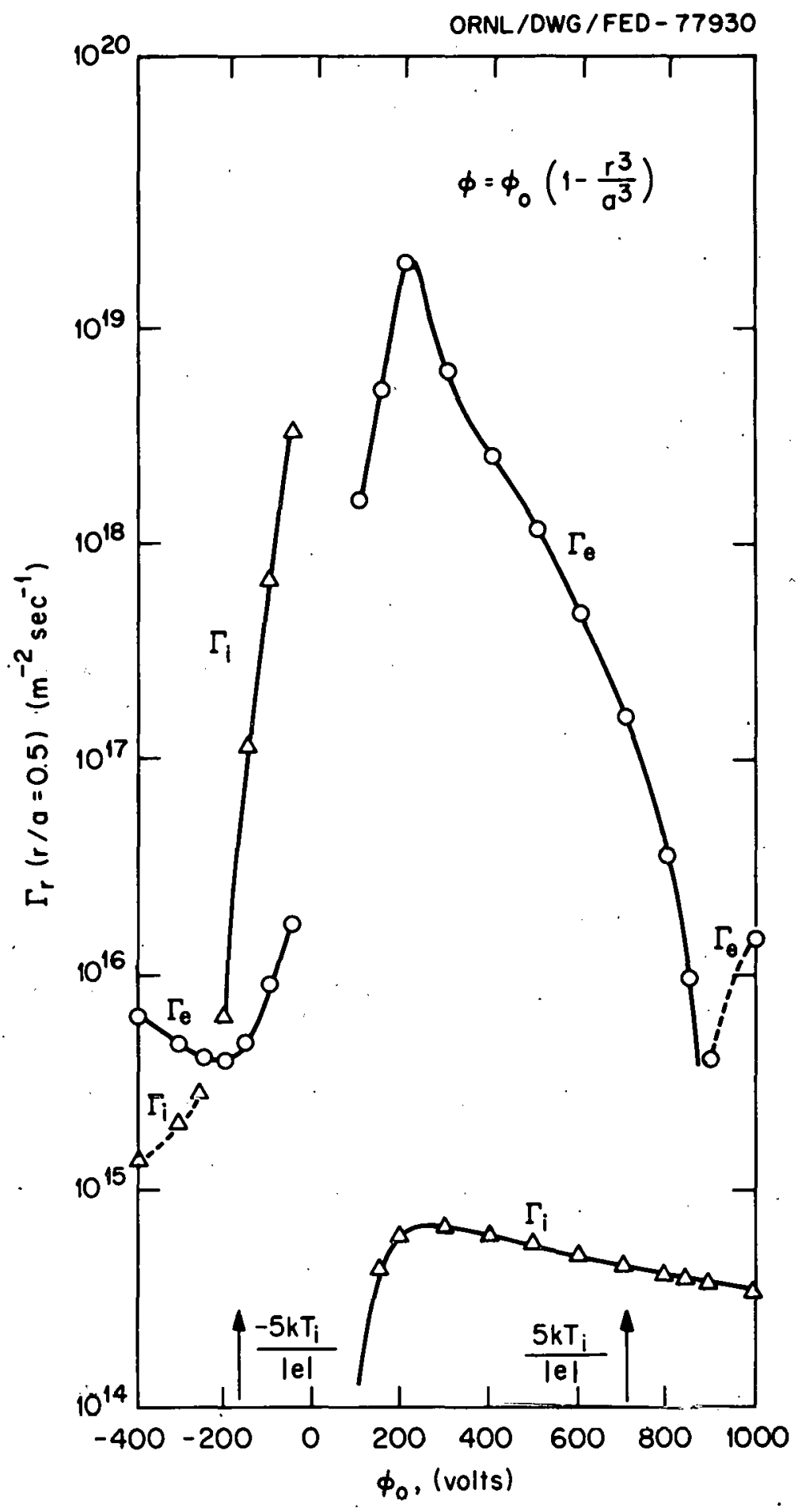

Fig. 6. Numerical results as in Fig. 3, but with a cubic potential profile. 
with $D_{n i} \approx \Gamma_{i} /\left(-\partial n / \partial r+n e E / k T_{i}\right)$. The dashed line shows the equivalent large field diffusion rate of Kovrizhnykh ${ }^{8}$ as used in zero-dimensional and one-dimensional transport models. Diffusion rates are normalized to $v_{o}^{2} /$ $\left(2<\Omega_{0}>\right)$ and plotted versus $\left|\nu_{i}\right|<\Omega_{0}>\mid$ where $\nu_{i}$ is the ion-ion collision frequency

$\nu_{i}=\frac{e^{4} n \ln \Lambda}{25.8 \sqrt{m_{i}^{\pi}} \varepsilon_{0}^{2}\left(k T_{i}\right)^{3 / 2}}$,

with $\Lambda=12 \pi\left(\varepsilon_{\mathrm{o}} \mathrm{kT}_{\mathrm{e}} / \mathrm{e}^{2}\right)^{3 / 2} / \mathrm{n}^{1 / 2}$. The velocity-averaged ion drift velocity from Eqs. (4) and (5) is

$<\Omega_{0}>=\frac{-k T_{i}}{e_{0} R_{c} r}-\frac{E}{B_{0} r}$.

For the parabolic potential assumed in Eq. (40), this gives an average cancellation of poloidal drifts, $<\Omega_{0}>=0$ in Eq. (41), which is uniform in radius for

$\phi_{0}=-\frac{k T_{i}}{\mid e T}\left(\frac{a}{R_{T}} \frac{N s}{2}\right)^{2} \approx-23 V$.

This accounts for the very large values of $\left.\left|\nu_{i}\right|<\Omega_{0}\right\rangle \mid$ in Fig. 5 near this potential. Also, the ion poloidal motion for negative potentials near the ion temperature is not sufficient to effectively average the vertical drift due to toroidal curvature, and an effective loss region forms in which ion orbits do not necessarily close within the machine. Ion diffusion rates are therefore enhanced in this case over those for positive potentials. This enhancement is shown in Fig. 5 tn he approximately two ordero of mag nitude for some negative potentials. For electrons, the situation is reversed and the enhanced loss region forms for positive potentials near the electron temperature

$\phi_{0} \approx \frac{k \mathrm{~T}}{\mathrm{e} T}\left(\frac{\mathrm{a}}{\mathrm{R}_{\mathrm{T}}} \frac{\mathrm{Ns}}{2}\right)^{2}$. 
perturbation in $\phi_{0}$ increases the electron diffusion rate, lowering pressure gradients, and leading to a net negative feedback on $\phi_{0}$.

It is tempting to guess that the differing stability properties of these two roots lead to a natural selection of the smaller negative root in the stable toroidal ( $T$ ) mode of operation and the larger positive root in the unstable mirror (M) mode of operation.

\section{CONCLUSIONS}

The calculations reported here show that inclusion of loss regions in velocity space where poloidal drifts cancel is essential to an accurate description of the self-consistent space potential in EBT. When such regions occur for ions near the center of the machine, radially inward pointing electric fields result with potentials a few times the ion temperature. In addition, the sign of the ambipolar field can play an important role in determining stability properties of the solution. In order to obtain a more accurate description of the evolution of the particle distributions calculated here, this work should be extended to include unperturbed distribution functions which are non-Maxwellian and which self-consistently reflect the loss rates calculated.

\section{ACKNOWLEDGMENT}

The authors wish to thank D. A. Spong, D. B. Batchelor, and the entire ORNL EBT experimental group for many helpful suggestions. 


\section{REFERENCES}

1. R. A. Dandl et al., Research Program for Plasma Confinement and Heating in ELMO Bumpy Torus Devices, ORNL/TM-4941, Oak Ridge National Laboratory, Oak Ridge, Tennessee (1975).

2. P. L. Colestock, EBT Group, Space Potential Profile Measurements on $E B T$, Technical Memo $\$ 77 / 47$ (1977), Oak Ridge National Laboratory, Oak Ridge, Tennessee.

3. c. L. Hedrick et al., A Simple Neoclassical Point Model for Transport and scaling in EBT, ORNL/TM-5490, Oak Ridge National Laboratory, Oak Ridge, Tennessee (1977); Nucl. Fusion 17 (6), 1237 (1977).

4. J. B. McBride and A. L. Sulton, Jr, LAPS-27/SAI-77-674-T.S, Laboratory for Applied Plasma Studies, Science Applications, Inc., La Jolla, California (1977).

5. E. F. Jaeger and C. L. Hedrick, Plasma Theory Section, Radial Transport with a Self-Consistent Ambipolar Electric Field in the ELMO Burqy Torus, Technical Memos \#77/47 (1977), Numerical Transport Predictions for EBT-S, $77 / 73$ (1977) and A Numerical Model for Radial Transport in the ELMO Bumpy Torus, ORNL/TM-6013 (1977), Oak Ridge National Laboratory, Oak Ridge, Tennessee; to be submitted to Nucl. Fusion.

6. R. D. Hazeltine, Plasma Phys. 15, 77 (1973).

7. D. A. Spong, E. G. Harris, and C. L. Hedrick, "Neoclassical Ion Transport Coefficients for the-ELMO Bumpy Torus Device," paper presented at the 18th Annual Meeting, Div. of Plasma Phys. of the American Physical Society, San Francisco, California, November 15-19, 1976; Bull. Am. Phys. Soc. 21, 1069 (1976) paper 3C-4.

8. L. M. Kovrizhnykh, Sov. Phys.-JETP 29, 475 (1969); and Preprint FIAN, No. 128, Mos cow (1969).

9. N. A. Krall and A. W. Trivelpiece, Principles of Plasma Physics, p. 316, McGraw-Hi11, New York, 1973.

10. D. C. Montgomery and D. A. Tidman, P1.rsmr Kinetice Theory, p. 34 , McGraw-Hill, New York, 1964.

11. C. F. Kennel and F. Engelmann, Phys. Fluids 9 , 2377 (1966).

12. 0. Eldridge, Phys. Fluids 15, 676 (1972).

13. J. Douglas and H. Rachford, Trans. Am. Math. Soc. 82, 421-439 (1956). 
ORNL/TM-6313

Dist. Category UC-20g

INTERNAL DISTRIBUTION

1. L. A. Berry
2. J. D. Callen
3. R. A. Dand1
4. R. A. Dory
5. G. G. Kelley
6. H. H. Haselton
7. P. N. Haubenreich
8. M. S. Lubell
9. O. B. Morgan
10. H. Postma
11. M. W. Rosenthal
12. J. Sheffield

\author{
13. D. Steiner \\ 14-45. E. F. Jaeger \\ 46. C. L. Hedrick \\ 47. J. S. Tolliver \\ 48-49. Laboratory Records Department \\ 50. Laboratory Records, ORNL-RC \\ - 51. Document Reference Section \\ 52-53. Central Research Library \\ 54. Fusion Energy Division Library \\ 55. Fusion Energy Division \\ Communications Center \\ 56. ORNL Patent Office
}

\section{EXTERNAL DISTRIBUTION}

57. Bibliothek, Max-Planck Institute für Plasmaphysik, 8046 Garching bei München, Federal Republic of Germany

58. Bibliothèque, Service du Confinement des Plasmas, C.E.A., B.P. No. 6, 92, Fontenay-aux Roses (Seine), France

59. Lung Cheung, Department of Electronics, University Science Center, The Chinese University of Hong Kong, Shatin, N.T., Hong Kong

60. J. F. Clarke, Office of Fusion Energy, G-234, Department of Energy, Washington, DC 20545

61. R. W. Conn, Fusion Technology Program, Nuclear Engineering Department, University of Wisconsin, Madison, WI 53706

62. CTR Library, c/o Alan F. Haught, United Technologies Research Laboratory, East Hartford, CT 06108

63. CTR Reading Room, c/o Allan N. Kaufman, Physics Department, University of California, Berkeley, CA 94720

64. J. Nar1 Davidson, School of Nuclear Engineering, Georgia Institute of Technology, Atlanta, GA 30332

65. Documentation S.I.G.N., Départment de la Physique du Plasma et de la Fusion Controlée, Association EURATOM-CEA sur la Fusion, Centre d'Études Nucléaires, B.P. 85, Centre du TRI, 38041 Grenoble, Cedex, France

66. W. R. Ellis, Office of Fusion Energy, G-234, Department of Energy, Washington, DC 20545

67. Harold K. Forsen, Exxon Nuclear Co., Inc., 777106 th Avenue, N.E., C-000777, Bellevue, WA 98009

68. Harold P. Furth, Princeton Plasma Physics Laboratory, Princeton University, Forrestal Campus, P.0. Box 451, Princeton, NJ 08540

69. Roy W. Gould, California Institute of Technology, Mail Stop 116-81, Pasadena, CA 91125

70. Robert L. Hirsch, Exxon Research and Engineering, P.0. Box 101, Florham Park, NJ 07932 
71. Raymond A. Huse, Manager, Research and Development, Public Service Gas and Electric Company, 80 Park Place, Newark, NJ 07101

72. T. Hsu, Office of Fusion Energy, G-234, Department of Energy, Washington, DC 20545

73. V. E. Ivanov, Physical-Technical Institute of the Ukranian Academy of Sciences, Sukhumt, U.S.S.R.

74. A. Kadish, Office of Fusion Energy, G-234, Department of Energy, Washington, DC 20545

75. L. M. Kovrizhnikh, Lebedev Institute of Physics, Academy of Sciences of the U.S.S.R., Leninsky Prospect 53, Moscow, U.S.S.R.

76. Guy Laval, Groupe de Physique Théorique, Ecole Polytechnique, 91 Palaiseau, Paris, France

77. Library, Centre de Recherches en Physique des Plasma, 21 Avenue des Bains, 1007, Lausanne, Switzerland

78. Library, Culham Laboratory, United Kingdom Atomic Energy Authority, Abingdon, Oxon, OX14 3DB, United Kingdom

79. Library, FOM-Institut voor Plasma - Fysica, Rijnhuizen, Jutphaas, Netherlands

80. Library, Institute for Plasma Physics, Nagoya University, Nagoya, Japan 464

81. Library, International Centre for Theoretical Physics, Trieste, Italy

82. Library, Laboratorio Gas Ionizzati, Frascati, Italy

83. Dsumber G. Lominadze, Academy of Sciences of the Georgian S.S.R., 8 Dzerzhinski St., 38004, Tbilisi, U.S.S.R.

84. Oscar P. Manley, Office of Fusion Energy, G-234, Department of Energy, Washington, DC 20545

85. D. G. McAlees, Exxon. Nuclear Co., Inc., Research and Technology Laser Enrichment Department, 2955 George Washington Way, Richland, WA 99352

86. J. E. McCune, School of Engineering, Department of Aeronautics and Astronautics, Bldg. 37-391, Massachusetts Institute of Technology, Cambridge, MA 02139

87. Claude Mercier, Service du Theorie des Plasmas, Centre d'Etudes Nucléaires, Fontenay-aux-Roses (Seine), France

88. K. G. Moses, Office of Fusion Energy, G-234, Department of Energy, Washington, DC 20545

89. D. Pfirsch, Institute for Plasma Physics, 8046 Garching be1 Munchen, Federal Republic of Germany

90. Plasma Physics Group, Department of Engineering Physics, Australian National University, P.O. Box 4, Canberra A.C.T. 2600, Australia

91. Robert E. Price, Office of Fusion Energy, G-234, Department of Energy, Washington, DC 20545

92. A. Rogister, Institute for Plasma Physics, KFA, Postfach 1913, D-5170, Jülich 1, Federal Republic of Germany

93. W. Sadowski, Office of Fusion Energy, G-234, Department of Energy, Washington, DC 20545

94. V. D. Shafranov, I. V. Kurchatov Institute of Atomic Energy, 46 Ulitsa Kurchatova, P.O. Box 3402, Moscow, U.S.S.R. 
95. Yu. S. Sigov, Institute of Applied Mathematics of the U.S.S.R. Academy of Sciences, Miuskaya, Sq. 4, Moscow A-47, U.S.S.R.

96. W. M. Stacey, Jr., School of Nuclear Engineering, Georgia Institute of Technology, Atlanta, GA 30332

97. J. B. Taylor, Culham Laboratory, United Kingdom Atomic Energy Authority, Abingdon, Oxon, OXI4 3DB, United Kingdom

98. Thermonuclear Library, Japan Atomic Energy Research Institute, Tokai, Naka, Ibaraki, Japan

99. Francisco Verdaguer, Director, Division of Fusion, Junta de Energia Nuclear, Madrid 3, Spain

100. Director, Research and Technical Support Division, Department of Energy, Oak Ridge Operations, P.O. Box E, Oak Ridge, TN 37830

101-276: Given distribution as shown in TID-4500, Magnetic Fusion Energy (Distribution Category UC-20g, Theoretical Plasma Physics) 